\title{
Texture Analysis of Brain CT Scans for ICP Prediction
}

\author{
Wenan Chen, Rebecca Smith, Nooshin Nabizadeh, Kevin Ward, \\ Charles Cockrell, Jonathan Ha, and Kayvan Najarian \\ Virginia Commonwealth University, \\ Richmond, Virginia 23221, United States
}

\begin{abstract}
Elevated Intracranial Pressure (ICP) is a significant cause of mortality and long-term functional damage in traumatic brain injury (TBI). Current ICP monitoring methods are highly invasive, presenting additional risks to the patient. This paper describes a computerized noninvasive screening method based on texture analysis of computed tomography (CT) scans of the brain, which may assist physicians in deciding whether to begin invasive monitoring. Quantitative texture features extracted using statistical, histogram and wavelet transform methods are used to characterize brain tissue windows in individual slices, and aggregated across the scan. Support Vector Machine (SVM) is then used to predict high or normal levels of ICP using the most significant features from the aggregated set. Results are promising, providing over $80 \%$ predictive accuracy and good separation of the two ICP classes, confirming the suitability of the approach and the predictive power of texture features in screening patients for high ICP.
\end{abstract}

Keywords: Computerized tomography (CT) images, Intracranial Pressure (ICP), texture analysis, Grey Level Run Length Method (GLRLM), Dual Tree Complex Wavelet Transform (DTCWT).

\section{Introduction}

One of the most severe complications after the initial TBI is increased intracranial pressure (ICP), typically resulting from edema caused by the original injury. Due to the rigid structure of the skull, elevated ICP can lead to shift and compression of soft brain tissue. Cerebral blood flow is proportional to the difference between the mean ICP in the brain and the mean arterial pressure. Therefore, as ICP increases, blood flow decreases, causing the brain to become ischemic and in turn leading to further brain damage due to lack of adequate blood flow 1]. ICP must therefore be closely monitored in the period after the initial injury [2. However, the standard method is highly invasive, involving cranial trepanation and the insertion of an ICP monitor device. This risks both infection and further damage to brain tissue. Consequently, a non-invasive screening method that can evaluate a patient's ICP level could potentially offer valuable assistance to physicians in deciding whether to perform trepanation.

A. Elmoataz et al. (Eds.): ICISP 2010, LNCS 6134, pp. 568 575, 2010.

(C) Springer-Verlag Berlin Heidelberg 2010 
Computed tomography (CT) scans are the standard image modality applied in TBI cases, due to their low cost and the low disruption they present to the patient. They also offer good definition of blood and bone matter. Currently, physicians often study CT scans of the brain when deciding whether to begin invasive ICP monitoring, looking for key signs such as brain midline shift or significant changes in the size of the lateral ventricles [3]. Both of these measures are quantifiable. However, there is no simple manual method to quantify changes in tissue texture, some of which may not be easily visible to the human eye. This has lead to interest in the computerized extraction of key texture features. Several prior studies have explored texture analysis of liver CT images in differentiating normal and malignant tissues. These have focused on the use of spatial domain statistical methods, including Spatial Grey Level Dependence Matrix (SGLDM) and Grey Level Run Length Method (GLRLM) 4156. Relevant to brain CTs, texture analysis has been used to automatically target radiotherapy for head-and-neck cancer based on CT images [7, and to distinguish between healthy tissue and intracerebral hemorrhage $(\mathrm{ICH})$ [ 8 . However, to the best of the authors' knowledge, texture analysis has not yet been utilized to provide quantitative features for predicting ICP.

This study focuses on the prediction of ICP levels using texture features extracted from brain CT scans via multiple processing methods. One possible pattern/regularity may be that with the increase of ICP, the density of the brain tissue increases because of the compression, which may result in the change in the appearance/texture of the CT images. Such possible patterns motivate the texture analysis on the CT images for ICP prediction. The method consists of four key stages: feature extraction from individual slices, aggregation across a full CT scan, feature selection, then classification via Support Vector Machine (SVM). A flow diagram of the approach is provided in Fig. 1.

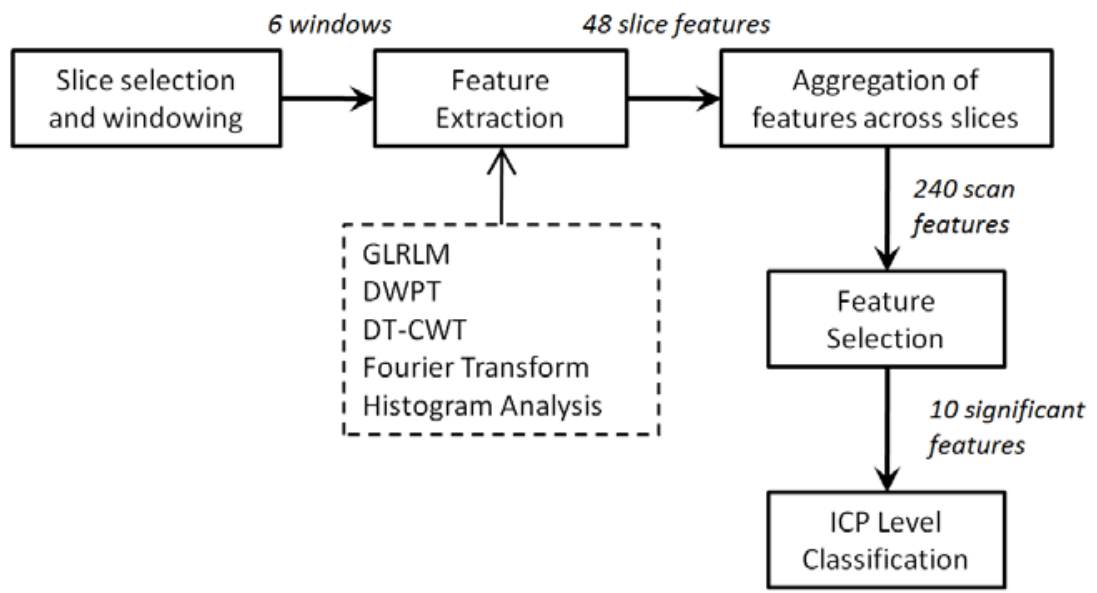

Fig. 1. Flow diagram of ICP prediction. The four key stages are shown, along with preliminary selection of slice windows to be analyzed. The five methods of feature extraction are also displayed. 


\section{Dataset and Labeling}

Fifty-six brain CT scans with a spatial resolution of 512x512 pixels and 8-bit grey level were provided by the Carolinas Healthcare System (CHS). These scans covered 17 patients diagnosed with mild to severe TBI. For each patient, ICP values were recorded every hour for each day they were monitored. Each CT scan is assigned an ICP score, calculated as the average of the two ICP readings closest to the time the scan was taken. The ICP scores for the scans are then grouped into two classes: high (ICP $>15 \mathrm{~mm} \mathrm{Hg})$ and normal $(\mathrm{ICP} \leq 15 \mathrm{~mm}$ $\mathrm{Hg}$ ), because $15 \mathrm{~mm} \mathrm{Hg}$ is usually the threshold distinguishing normal ICP level and elevated ICP level used by physicians. Using this threshold, the dataset contains 31 normal ICP cases and 25 with high ICP. Five slices covering the region expected to contain ventricle matter were manually selected from each scan for analysis.

\section{Methodology}

\subsection{Texture Feature Extraction}

Six rectangular windows are extracted from each of the five selected CT slices; these are selected to contain primarily neural tissue. Figure 2 illustrates the chosen regions. Each window is processed separately, with a total of 48 texture features being extracted using five methods: Grey Level Run Length Matrix (GLRLM), Histogram analysis, Fourier Transform, Discrete Wavelet Packet Transform (DWPT), and Dual Tree Complex Wavelet Transform (DT-CWT). Although not all of these features may be useful in the final classification task, the feature selection step removes those considered irrelevant. Furthermore, since this method is ultimately intended to provide a generalized framework for texture-based classification of CT scans, these additional measures may prove useful in analysis tasks involve other types of tissue elsewhere in the body.

Grey Level Run Length Method (GLRLM). A grey-level run can be considered a set of consecutive collinear pixels that share the same grey level value. These runs characterize texture elements in images, and each one is defined by its direction, length, and grey level value [9. Four directions are examined in this study $(0,45,90$ and 135 degrees) and the runs for each are used to construct four separate run-length matrices. The element $P(i, j)$ of a run-length matrix $P$ reflects the number of runs $j$ of pixels with gray level value $i$ in the specified direction for the matrix. $P$ is size $(n \times k)$, where $n$ is the maximum grey level and $k$ is the maximum possible run length in the image being analyzed. Eleven features are then calculated from each of the four run matrices: Short Run Emphasis (SRE), Long Run Emphasis (LRE), Grey Level Nonuniformity (GLN), Run Length Nonuniformity (RLN), Run Percentage (RP), Low GrayLevel Run Emphasis (LGRE), High Gray-Level Run Emphasis (HGRE), Short Run Low Gray-Level Emphasis (SRLGE), Short Run High Gray-Level Emphasis (SRHGE), Long Run Low Gray-Level Emphasis (LRLGE), and Long Run High 


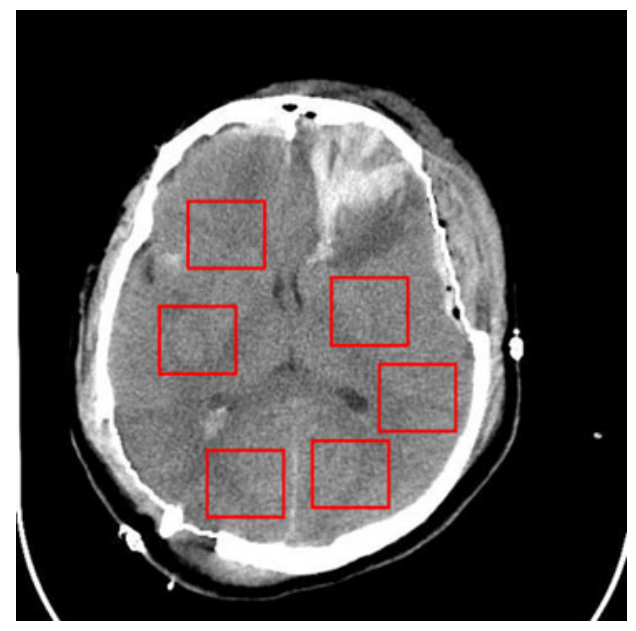

Fig. 2. Location of the six windows used for texture extraction

Gray-Level Emphasis (LRHGR). These provide a statistical characterization of image texture based on both gray-level and run-length that can be used as input to the classification process. Their formulations can be found in [10].

Histogram Analysis. Four features are obtained from the histogram for each window: average intensity level, average contrast, smoothness and entropy. The first two measures are respectively defined as the histogram's mean and standard deviation. A measure of smoothness is provided by

$$
R=1-\frac{1}{1+\sigma^{2}}
$$

where $\sigma$ is the standard deviation. A value approaching 0 indicates low variation in pixel value intensity (i.e. smooth texture), while a value approaching 1 shows much higher variation (i.e. rough texture). The final feature, histogram entropy, reflects the randomness of intensity values.

Fourier Features. Fast Fourier Transform (FFT) is applied to each window. The maximum, minimum, average and median of the resulting frequencies are then recorded as texture features, along with the frequency corresponding to the median value of the absolute value.

Discrete Wavelet Packet Transform (DWPT). The standard Discrete Wavelet Transform (DWT) decomposes a signal by passing it through a series of filters at consecutive levels of decomposition. At each level $i$, both a high-pass and low-pass filter are applied to the signal passed from level $i-1$, extracting detail and approximation coefficients respectively. Only the approximation coefficients are then passed on to the next level $i+1$. In image processing, this creates four new sub-images at each level by sub-sampling the input by 2 . This 
multi-resolution analysis is much more effective in detecting localized texture patterns than FFT's global approach. The Discrete Wavelet Packet Transform (DWPT) extends DWT by also decomposing the detail coefficients at each level [11. Though this incurs a higher computational cost, it provides a richer representation of texture which is valuable to the task in this study.

Dual Tree Complex Wavelet Transform (DT-CWT). Dual tree complex wavelet transform 12 is designed to overcome some shortcomings when applying Discrete Wavelet Transform (DWT) in higher dimension (e.g., image processing). There exist problems in DWT such as oscillations around sigularity, shift variance, aliasing after processing on wavelet coefficients, and lack of directionality. However, these problem do not happen in Fourier Transform. DTCWT tries to deal with these issues by adopting some characters from the Fourier Transform while keeping the wavelet advantage in time-frequency analysis. In image processing applications, this method is known to be free of checker board artifact in DWT and to provide 6 directional wavelets in the $2 \mathrm{D}$ dimension.

The reason that Frourier Transform avoid those problems above is that it is in the complex domain and that the real part and imaginary part form a Hilbert Transform pair. In DTCWT, the scaling function and wavelet function are also complex valued. For example, the wavelet function can be expressed as:

$$
\psi_{c}(t)=\psi_{r}(t)+j \psi_{i}(t)
$$

where $\psi_{r}(t)$ is the real-valued function for the real part and $\psi_{i}(t)$ is the realvalued function for the imaginary part. In the DTCWT, the real and imaginary parts are processed separatly using a DWT scheme.

The advantage of DTCWT in texture analysis is that it makes the location of texture patterns not sensitive. Also it can capture more directions of texture patterns directly from the image. In this application, each subimage for texture analysis is decomposed into the first level, then the energy of each subband is calculated as in DWT part above, the entropy of energy distribution is also calculated as in DWT part.

\subsection{Slice Aggregation and Feature Selection}

Since ICP labeling is done on a full scan basis, it is necessary to combine the features extracted from each of the scan slices to create a new aggregated feature set to be used in classification. For a given slice, the features for the six windows are averaged, giving a total of 48 features per slice. For any given texture feature $f$, five new aggregative features are calculated across all slices: $\min (f), \max (f)$, $\operatorname{median}(f), \mu(f)$, and $\sigma(f)$. This gives a total of $48^{*} 5=240$ features for the entire scan. This simplified approach is taken to save computational time and to circumvent the issue of large gaps between slices.

Feature selection is then applied to the aggregated feature set to remove irrelevant features and improve performance. This is done via a two-stage filter-wrapper 
approach. In the first stage, the ReliefF algorithm [13] is used to rank the features in terms of their significance. The top 50 are then passed to the second stage. In the second stage, the Support Vector Machine (SVM) is used in the wrapper for the classification of feature set. In the validation step, the 10 fold cross-validation result is used as the accuracy measurement. Radical Basis Function (RBF) kernel is used with fixed parameters of $C=32768$ and $\gamma=0.125$. The search method used to construct feature set is Best First [16]. The feature subset achieving the best validation accuracy for ICP prediction is selected as the final feature set, which contains 10 features in the set. All feature selection steps are performed in the Weka machine learning software [14].

\subsection{Classification}

Classification is performed using the Support Vector Machine (SVM) algorithm, which is widely used in classification tasks and typically offers good accuracy. To accommodate the relatively low number of scans in the dataset, 10-fold crossvalidation is used to measure performance.

\section{Results}

The result of the proposed method is evaluated using the following criterion: sensitivity, specificity and accuracy [15. Sensitivity is defined as

$$
\text { sensitivity }=\frac{\text { number of True Positives }}{\text { Total number of Positives }}
$$

while specificity is defined as

$$
\text { specificity }=\frac{\text { number of True Negatives }}{\text { Total number of Negatives }}
$$

The classification results are provided in Table 1 It can be seen that the designed ICP prediction method offers very good accuracy, despite the relatively small size of the dataset. Sensitivity and specificity are also good, meaning that the two classes - normal and high ICP - are well distinguished. These results indicate that texture analysis alone has the potential to be highly valuable in a non-invasive ICP screening method.

Table 1. Classification results using SVM

\begin{tabular}{lll}
\hline Sensitivity & Specificity & Accuracy \\
\hline $82.25(+-1.74)$ & $81.20(+-0.04)$ & $81.79(+-2.30)$ \\
\hline
\end{tabular}




\section{Conclusions}

This paper provides a non-invasive screening method for ICP prediction based on quantitative texture features extracted from CT brain scans. Feature extraction methods used include histogram analysis, wavelet transform and statistical gray level analysis. These are applied to windows in each slice, and the features aggregated across the entire CT scan. By selecting the most informative features for the classification task, a promising ICP prediction model is built. Due to the number of features extracted, the approach can potentially form a framework for analysis of other body regions; furthermore, new methods of texture analysis can easily be incorporated. Once refined, the method may thus be suitable for incorporation as a module into a wider TBI decision-support system. Future work will involve combining texture analysis with the authors' existing work on evaluation of mid-line shift, ventricle size and blood volume, with the hope of further improving prediction accuracy. The method will also be tested on a large dataset, possibly allowing the selection of a larger feature set for classification. Finally, the medical interpretations of various texture features will be explored in more depth with the help of physicians.

\section{Acknowledgments}

The authors would like to acknowledge the Carolinas Healthcare System (CHS) for providing the CT dataset and ICP recordings.

\section{References}

1. Koch, M.A., Narayan, R.K., Timmons, S.D.: Traumatic Brain Injury. In: Merck manual online, http://www.merck.com/mmpe/sec21/ch310/ch310a.html (retrieved)

2. Castellano, G., Bonilha, L., Li, L.M., Cendes, F.: Texture Analysis of Medical Images. Clinical Radiology 59(12), 1061-1069 (2004)

3. Sucu, H.K., Gelal, F., Gokmen, M., Ozer, F.D., Tektas, S.: Can midline brain shift be used as a prognostic factor to predict postoperative restoration of consciousness in patients with chronic subdural hematoma? Surgical Neurology 66(2), 178-182 (2006)

4. Chen, E.L., Chung, P., Chen, C.L., Tsa, H.M., Chang, C.I.: An automated diagnostic system for CT liver image classification. IEEE Trans. Biomed. Eng. 45(6), 783-794 (1998)

5. Mir, A.H., Hanmandlu, M., Tandon, S.N.: Texture Analysis of CT Images. IEEE Eng. Med. Biology 14, 781-786 (1995)

6. Mougiakakou, S., Valvanis, I., Nikita, K.S., Nikita, A., Kelekis, D.: Characterization of CT liver lesions based on texture features and a multiple neural network classification scheme. In: 25th Int. Conf. of the IEEE Engineering in Medicine and Biology Society, vol. 2, pp. 1287-1290 (2003)

7. Yu, H., Caldwell, C., Mah, K., Poon, I., Balogh, J., MacKenzie, R., Khaouam, N., Tirona, R.: Automated Radiation Targeting in Head-and-Neck Cancer Using Region-Based Texture Analysis of PET and CT Images. International Journal of Radiation Oncology*Biology*Physics 75(2), 618-625 (2009) 
8. Kabara, S., Gabbouj, M., Dastidar, P., Alaya-Cheikh, F., Ryymin, P., Lassonen, E.: CT Image Texture Analysis of Intracerebral Hemorrhage. In: 2003 Finnish Signal Processing Symposium (FINSIG'03), Tampere, Finland, pp. 190-194 (2003)

9. Chu, A., Sehgal, C.M., Greenleaf, J.F.: Use of gray value distribution of run lengths for texture analysis. Pattern Recognition Letters 11(6), 415-420 (1990)

10. Tang, X.: Texture information in run-length matrices. IEEE Trans. Image Proc. 7(11), 1602-1609 (1998)

11. Manthalkar, R., Biswas, P.K., Chatterji, B.N.: Rotation and scale invariant texture features using discrete wavelet packet transform. Pattern Recognition Letters 24(14), 2455-2462 (2003)

12. Kingsbury, N.: Complex Wavelets for Shift Invariant Analysis and Filtering of Signals. Applied and Computational Harmonic Analysis 10(3), 234-253 (2001)

13. Robnik-Sikonja, M., Kononeko, I.: An adaptation of relief for attribute estimation in regression. In: ICML'97: 14th International Conference on Machine Learning, pp. 296-304 (1997)

14. Weka 3: Data Mining Software in Java, http://www.cs.waikato.ac.nz/ml/weka/

15. Altman, D.G., Bland, J.M.: Diagnostic tests. 1: Sensitivity and specificity. British Medical Journal 308 (6943), 1552 (1994)

16. Ron, K., John George, H.: Wrapper for feature subset selection. Artificial Intelligence 97(1-2), 273-324 (1997) 